\title{
A cadaveric study investigating the anatomy of the medial orbital wall
}

\author{
Kathylin M. Hester ${ }^{1}$, Omid B. Rahimi ${ }^{1}$, Constance L. Fry ${ }^{2}$, Haley L. Nation ${ }^{1}$ \\ ${ }^{1}$ Department of Cell Systems and Anatomy, UT Health San Antonio, San Antonio, TX, ${ }^{2}$ Department of Ophthalmology, UT Health San Antonio, San \\ Antonio, TX, USA
}

\begin{abstract}
The purpose of this study is to investigate the applicability of the current surgical guideline, known as '24-12-6' surgical guideline, in the Hispanic and European populations. This guideline is used during numerous orbital surgeries and states that the distance between the anterior lacrimal crest (ALC) to the anterior ethmoidal foramen (AEF) (24 mm), the AEF to the posterior ethmoidal foramen (PEF) $(12 \mathrm{~mm}$ ), and the PEF to the optic canal (OC) $(6 \mathrm{~mm})$ follows a Rule of Halves. Previous studies suggest this surgical guideline is not applicable for all ethnicities; however, to our knowledge, no data has been published regarding the accuracy of this guideline pertaining to the Hispanic population. An experimental study was performed on 79 orbits (52 cadavers) donated to the Human Anatomy Program at UT Health San Antonio. The ALC, AEF, posterior ethmoidal foramen, and OC were identified; the orbit was enucleated and all remaining soft tissue removed. The distance between each landmark was recorded using a digital caliper. For all cadavers studied, the distances between the ALC, AEF, posterior ethmoidal foramen, and OC were $24.76 \mathrm{~mm}, 13.89 \mathrm{~mm}$, and $7.61 \mathrm{~mm}$, respectively. Thus, the '24-12-6' surgical guideline was not applicable to the sample studied. Based on ethnicity data, these relationships were also not true for the European or the Hispanic populations. Therefore, significant anatomical variations exist in the current surgical guideline. Clinicians may need to adjust their methodology during surgical procedures in order to optimize patient care.
\end{abstract}

Key words: Medial orbital wall, Orbit anatomy, 24-12-6 mm surgical guideline, Rule of halves

Received January 29, 2021; Revised March 8, 2021; Accepted March 11, 2021

\section{Introduction}

Numerous clinical procedures require detailed knowledge of the medial orbital wall anatomy. Examples of such procedures include anterior and posterior ethmoidal vessel ligations for epistaxis control, orbital decompression to treat thyroid exophthalmos, medial wall reconstruction after resection of sino-orbital neoplasms and infections, and surgical repair of medial wall fractures after periorbital trauma.

\section{Corresponding author:}

Haley L. Nation (iD)

Department of Cell Systems and Anatomy, UT Health San Antonio, San Antonio, TX 78229, USA

E-mail: Nation@uthscsa.edu
These procedures account for a high percentage of surgeries performed by ophthalmologists and other health care providers. While performing these procedures, knowledge of detailed skeletal characteristics of the medial orbital wall is necessary to avoid irreparable damage to nerves and vasculature, extraocular muscles paralysis, cerebrospinal fluid leakage, and blindness [1].

The anatomical relationship that describes the distance between the anterior lacrimal crest (ALC) to the anterior ethmoidal foramen (AEF), the AEF to the posterior ethmoidal foramen (PEF), and the PEF to the optic canal (OC), is known as the '24-12-6' surgical guideline or the Rule of Halves. Though the exact origin of this rule is unknown, a study conducted by Rontal et al. [2] in 1979 examined skulls of Indian origin and found that the measurements from the ALC to the AEF was $24 \mathrm{~mm}$, AEF to PEF was $12 \mathrm{~mm}$, and

\section{Copyright (c) 2021. Anatomy \& Cell Biology}

This is an Open Access article distributed under the terms of the Creative Commons Attribution Non-Commercial License (http://creativecommons.org/licenses/by-nc/4.0/) which permits unrestricted non-commercial use, distribution, and reproduction in any medium, provided the original work is properly cited. 
PEF to OC was $6 \mathrm{~mm}$. Additional mention of the '24-12-6' surgical guideline was published in a 1992 paper regarding epistaxis [3].

Previous reports have suggested this '24-12-6' mm guideline may not accurately represent all populations. Anatomical variations in the medial orbital wall exist between British Caucasian, African American, Chinese, Korean, and Thai people [4-8]. It is evident that numerous studies have been conducted on the bony orbital dimensions in various populations and recommended that the '24-12-6' mm relationship be used as a guideline rather than a rule.

The Hispanic population accounted for more than half (52\%) of the United States' population growth between 2010-2019 [9]. In 2019, the U.S. Hispanic population reached a record 60.6 million, or $18 \%$ of the U.S. population [9]. To our knowledge, the relationships under investigation have not been studied in the Hispanic population. Therefore, in order to bridge this gap of knowledge, we examined the medial orbital walls of Hispanic cadavers to determine if there is a significant difference between these critical landmarks compared to a European population and to the commonly held guideline.

\section{Materials and Methods}

The design of this study was a cadaveric investigation examining 52 adult cadavers. Body donors used in this study were gifted to the Human Anatomy Program at the UT Health San Antonio. UT Health San Antonio is a member institution of the State Anatomical Board of Texas. All donations to the program are registered and cared for under the statutes and guidelines of the State of Texas. Methods and protocols involved in this study have been approved through the program. No IRB approval was required as the project was deemed not regulated research (protocol number: HSC20180149N).

Cadavers used in this project were previously dissected by dental students over the 2017-2019 academic years. The cadaver population studied consisted of 27 females and 25 males (determined by external genitalia); the average age of all cadavers was 81.6 (minimum $=55$ years, maximum $=98$ years). Age, ethnicity, and cause of death were obtained through family and funeral reports. The term Hispanic is used to describe people who were reported as Hispanic, Mexican, Mexican-American or South American. Only cadavers who were either Hispanic or European were included in the study. Reasons for exclusion from the study were skulls in which the bony landmarks of interest were not intact.

Using a scalpel, the eyelid and any other connective tissue spanning from the superciliary arch to the infraorbital rim was reflected and removed to gain access to the ocular globe. The orbit was enucleated and all remaining soft tissue (muscles, vessels, nerves, and periorbita) was removed with forceps and a periorbita scraper. All critical landmarks were identified: these included the ALC, the AEF, the PEF, the OC, and any additional ethmoidal foramina along the medial wall of the orbit. Structures traversing the ethmoidal foramina and OC were left intact if the anterior and posterior edge of the foramen was visible, otherwise traversing structures were removed. Using a 26-gauge flexible wire, distances outlined by the '24-12-6' guideline were measured by marking the wire and measuring the marked wire with a Vernier caliper (Scienceware; Bel-Art Products, Wayne NJ, CAT No. 134150000) to the nearest $0.1 \mathrm{~mm}$.

The first measurement taken was from the midpoint (approximated) of the ALC to the anterior edge of the AEF (Fig. 1). The second measurement was from the posterior aspect of the AEF to the anterior edge of the PEF. The third measurement was taken from the posterior edge of the PEF to the medial edge of the OC. Measurements were taken by two individuals and the average distance was calculated. If interobserver variability was greater than $1.0 \mathrm{~mm}$, each researcher re-measured the distance in question and the aver-

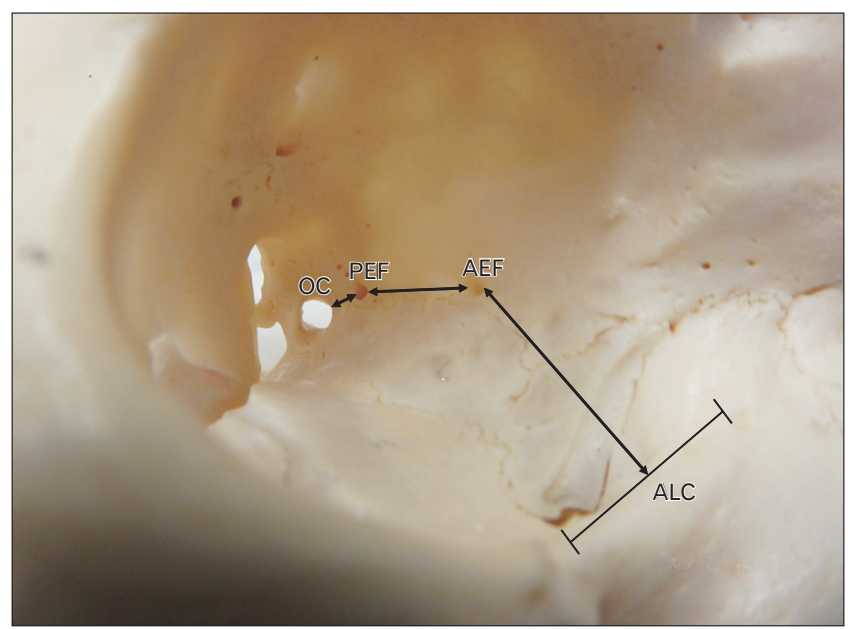

Fig. 1. Surgical guideline measurements. The current surgical guideline approximates the distances between the ALC, AEF, PEF, and OC. Right orbit. AEF, anterior ethmoidal foramen; ALC, anterior lacrimal crest; OC, optic canal; PEF, posterior ethmoidal foramen. 
age of the three closest measurements was calculated.

\section{Results}

\section{Comparison to surgical guideline}

Data from all measurements $(n=79)$, regardless of sex, side of the face, and ethnicity, were analyzed. A one-sample t-test and Wilcoxon test with a $95 \%$ confidence interval (CI) was performed to investigate whether the ALC-AEF, AEF-PEF, and PEF-OC medial orbital wall measurements adhered to the current surgical guideline. There was a statistically significant difference $(P=0.0019)$ between the ALC-AEF measurement $(24.76 \mathrm{~mm})$ and the $24 \mathrm{~mm}$ distance allotted by the surgical guideline. Likewise, there was a statistically significant difference $(P<0.0001)$ between the AEF-PEF measurement $(13.89 \mathrm{~mm})$ and the $12 \mathrm{~mm}$ surgical guideline. Lastly, there was a statistically significant difference $(P<0.0001)$ between the PEF-OC measurement $(7.614 \mathrm{~mm})$ and the $6 \mathrm{~mm}$ surgical guideline.

In addition to comparing all cadavers studied to the surgical guideline, each population, European and Hispanic, was separately compared to the surgical guideline to determine whether the accuracy of this guideline depended on ethnicity (Fig. 2). A one-sample t-test and Wilcoxon test was performed to compare the European sample ( $\mathrm{n}=70$ orbits) to the surgical guideline (Fig. 2). There was a statistically sig-

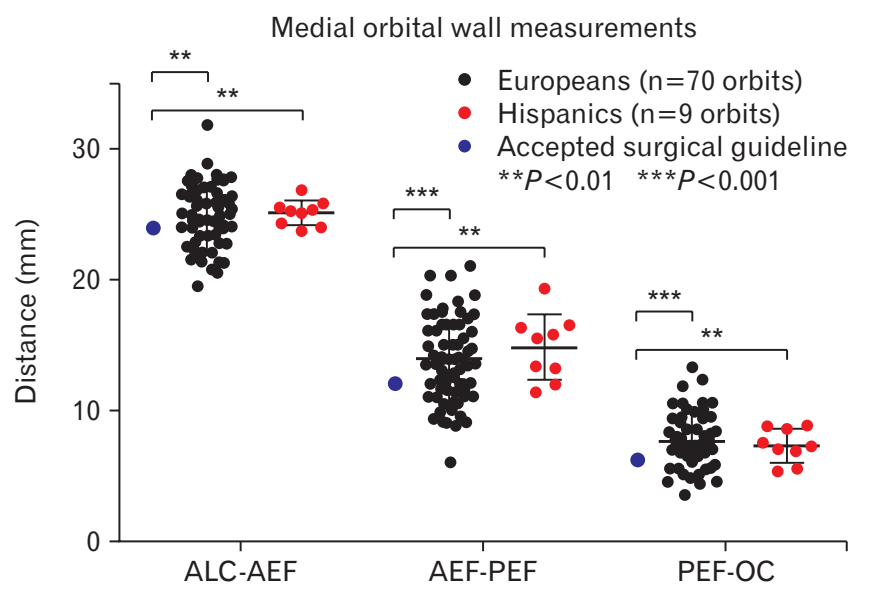

Fig. 2. Medial orbital wall Measurements Based on Populations. There was a statistically significant difference in medial orbital wall measurements between European and Hispanic samples when compared to the accepted surgical guideline. There was no statically significant difference in medial orbital wall measurements between ethnicities. AEF, anterior ethmoidal foramen; ALC, anterior lacrimal crest; OC, optic canal; PEF, posterior ethmoidal foramen. nificant difference in the ALC-AEF measurement $(P=0.0079$, $24.72 \mathrm{~mm}$ vs. $24 \mathrm{~mm})$, the AEF-PEF $(P<0.001,13.78 \mathrm{~mm}$ vs. $12 \mathrm{~mm})$ and the PEF-OC $(P<0.0001,7.53 \mathrm{~mm}$ vs. $6 \mathrm{~mm})$ measurement compared to the surgical guideline. A onesample t- test and Wilcoxon test was also performed to compare the Hispanic sample ( $\mathrm{n}=9$ orbits) to the surgical guideline (Fig. 2).There was a statistically significant difference in the ALC-AEF measurement $(P=0.0104,25.07 \mathrm{~mm}$ vs. 24 $\mathrm{mm})$, the AEF-PEF measurement $(P=0.0108 ; 14.80 \mathrm{~mm}$ vs. $12 \mathrm{~mm})$, and the PEF-OC measurement $(P=0.0120,8.30 \mathrm{~mm}$ vs. $6 \mathrm{~mm}$ ) compared to the surgical guideline. Therefore, the surgical guideline did not hold true for either the European or Hispanic populations.

\section{Comparison between ethnicities}

To determine if there was a statistically significant difference between the medial orbital wall measurements in European $(\mathrm{n}=70)$ and Hispanic $(\mathrm{n}=9)$ samples, an unpaired t-test was performed (Fig. 2). There was no statistically significant difference between the European and Hispanic samples in the ALC-AEF measurement $(P=0.6494,24.72 \mathrm{~mm}$ vs. $25.07 \mathrm{~mm})$, AEF-PEF measurement $(P=0.3265,13.78 \mathrm{~mm}$ vs. $14.80 \mathrm{~mm})$, or PEF-OC measurement $(P=0.2336,7.53 \mathrm{~mm}$ vs. $8.30 \mathrm{~mm})$. In conclusion, for all medial orbital wall measurements, there was no statistically significant difference between the European versus the Hispanic sample.

\section{Correlation between measurements}

To determine if the length of one measurement can predict the other two measurements, a Pearson r-correlation test was performed. There was a modest negative linear relationship ( $\mathrm{r}=-0.2977 ; P=0.012)$ between the ALC-AEF and AEFPEF. Meanwhile, there was a stronger negative relationship $\left(\mathrm{r}=-0.4907 ; P=1.617 \times 10^{-5}\right)$ between the AEF-PEF and PEFOC measurements. Finally, there was a weak positive linear relationship ( $\mathrm{r}=0.1714 ; P=0.156)$ between the ALC-AEF and PEC-OC measurements. Thus, while not all measurements can be used to accurately predict the remaining relationships, the AEF-PEF distance significantly influences the PEF-OC measurement.

\section{Interaction between sex and side of the face}

Lastly, a two-way-ANOVA mixed effect analysis was performed to account for possible interaction between random or non-random variables, (e.g., sex and left vs. right sides of the face) and account for missing values (e.g., unilateral 
samples).

The mixed-effect analysis for the ALC-AEF measurement yielded that sex (male vs. female) had no effect or interaction $(P=0.8730)$ with the data (Fig. 3A). Therefore, the male and female samples were combined; the left (mean=25.18 $\mathrm{mm}$ ) versus right $($ mean $=24.44 \mathrm{~mm}$ ) orbit did have a statistically significant effect $(P=0.0190)$ on the ALC-AEF measurement (Fig. 3B). The data indicates that for left orbits, the 24 $\mathrm{mm}$ surgical guideline falls below the 95\% CI while for the right orbit the $24 \mathrm{~mm}$ surgical guideline falls within the
95\% CI. In fact, $82.9 \%$ of left orbits and $63.6 \%$ of right orbits contained ALC-AEF measurements that were at or above the recommended surgical guideline. Meanwhile, and more important clinically, $17.1 \%$ of left orbits and $36.4 \%$ of right orbits contained ALC-AEF measurements that were below the recommended surgical guideline.

The mixed-effect analysis for the AEF-PEF measurement yielded that while the left versus right orbit had no effect or interaction $(P=0.1232)$ on the AEF-PEF measurement (Fig. $4 \mathrm{~A})$, sex did have a statistically significant effect $(P=0.0253)$
A

ALC-AEF

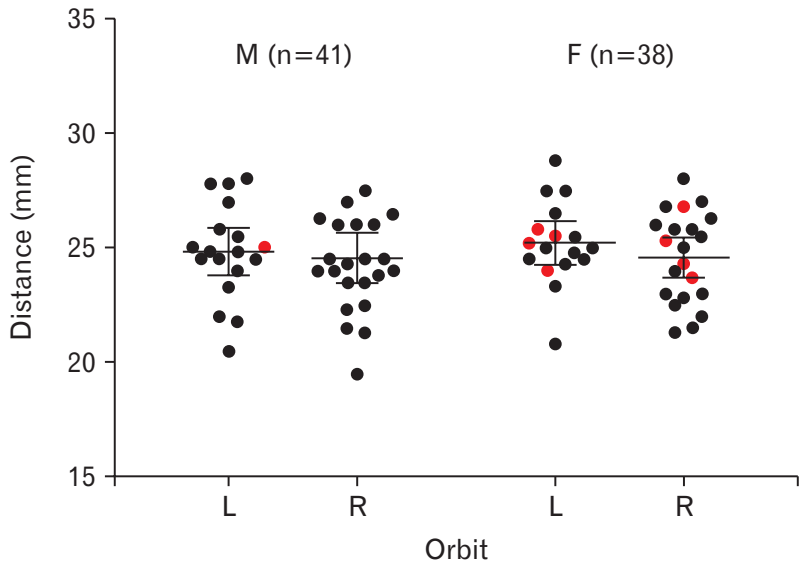

B

ALC-AEF (M/F combined)

- Europeans ( $n=70$ orbits)

- Hispanics ( $n=9$ orbits)

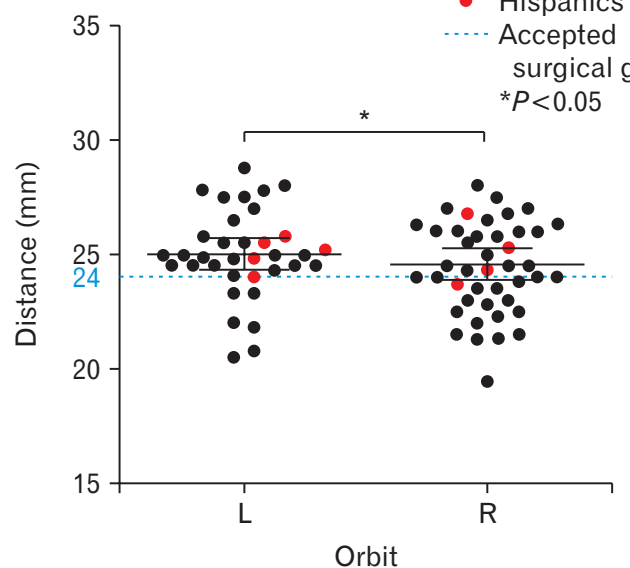

Fig. 3. ALC-AEF measurements between sex and side of the body. While there was no statistically significant difference in ALC-AEF measurements between male and female samples (A), there was a statistically significant difference between the left and right sides of the face (B). AEF, anterior ethmoidal foramen; ALC, anterior lacrimal crest; F, female; L, left; M, male; R, right.

A

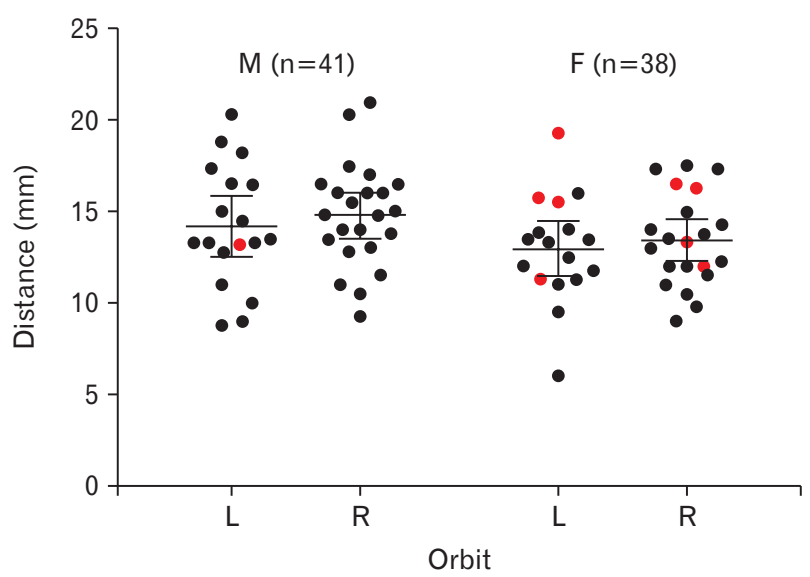

B

AEF-PEF (L/R combined)

- Europeans ( $n=70$ orbits)

- Hispanics ( $n=9$ orbits)

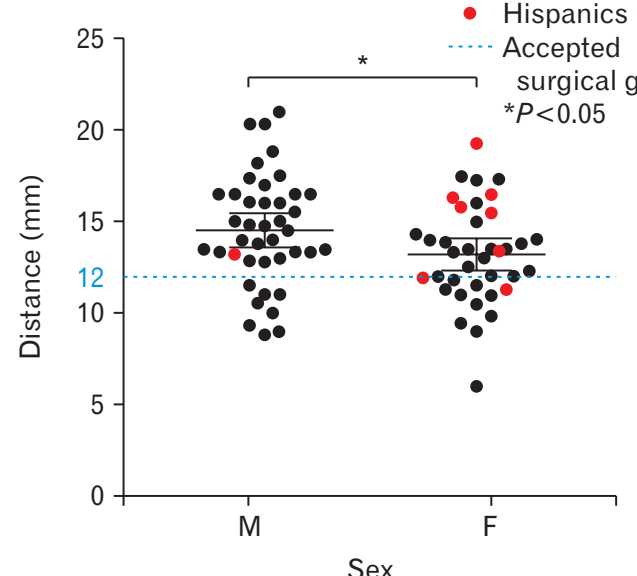

Sex

Fig. 4. AEF-PEF measurements between sex and side of the body. While there was no statistically significant difference in AEF-PEF measurements between left and right sides of the face (A), there was a statistically significant difference between male and female samples (B). AEF, anterior ethmoidal foramen; F, female; L, left; M, male; PEF, posterior ethmoidal foramen; R, right. 
A

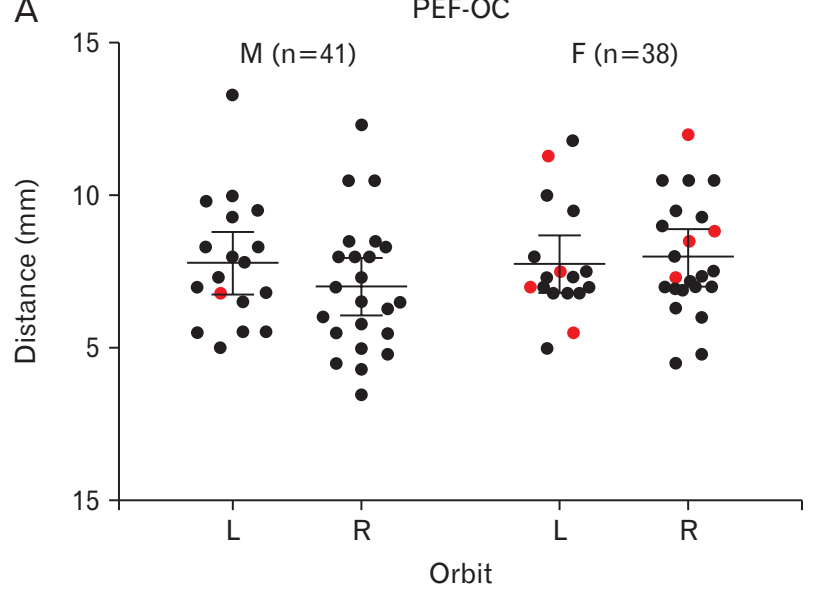

B

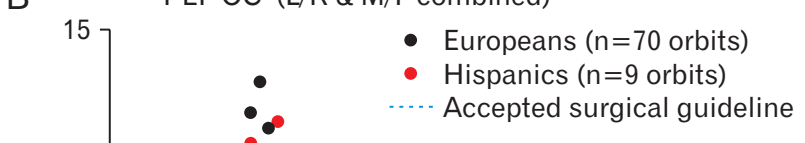

Fig. 5. PEF-OC measurements between sex and side of the body. There was no statistically significant difference in PEF-OC measurements between left and right sides of the face or between male and female samples. F, female; L, left; M, male; OC, optic canal; PEF, posterior ethmoidal foramen; R, right.

on the measurement (Fig. 4B). The data collected illustrate that for males and females, the $12 \mathrm{~mm}$ surgical guideline falls below the $95 \%$ CI. $71.4 \%$ of left orbits and $79.5 \%$ of right orbits contained AEF-PEF measurements that were at or above the recommended surgical guideline. Meanwhile, and more important clinically, $28.6 \%$ of left orbits and $20.5 \%$ of right orbits contained AEF-PEF measurements that were below the recommended surgical guideline.

The mixed-effect analysis for the PEF-OC measurement yielded that orbital side $(P=0.2724)$ and $\operatorname{sex}(P=0.5325)$ had a no statistically significant effect on the data (Fig. 5A). Therefore, male/female and left/right variables were compiled; the $6 \mathrm{~mm}$ surgical guideline fell below the $95 \%$ CI (Fig. 5B). $82.9 \%$ of left orbits and $77.3 \%$ of right orbits contained PEFOC measurements that were at or above the recommended surgical guideline. Meanwhile, and more important clinically, $17.1 \%$ of left orbits and $22.7 \%$ of right orbits contained PEF-OC measurements that were below the recommended surgical guideline.

\section{Additional findings}

Numerous medial orbital walls anatomically deviated from the classical description (one AEF and one posterior ethmoidal foramen). First, our study found that 11/79 orbits (13.9\%) had a 'middle ethmoidal foramen' located between the AEF and PEF. Comparatively, studies reported anywhere from $16.5 \%$ to $39.9 \%$ of orbits with a middle ethmoidal foramina $[1,2,4,6,8,10]$. Another interesting finding was that $3 / 79$ orbits $(3.8 \%)$ had a missing PEF and thus only had

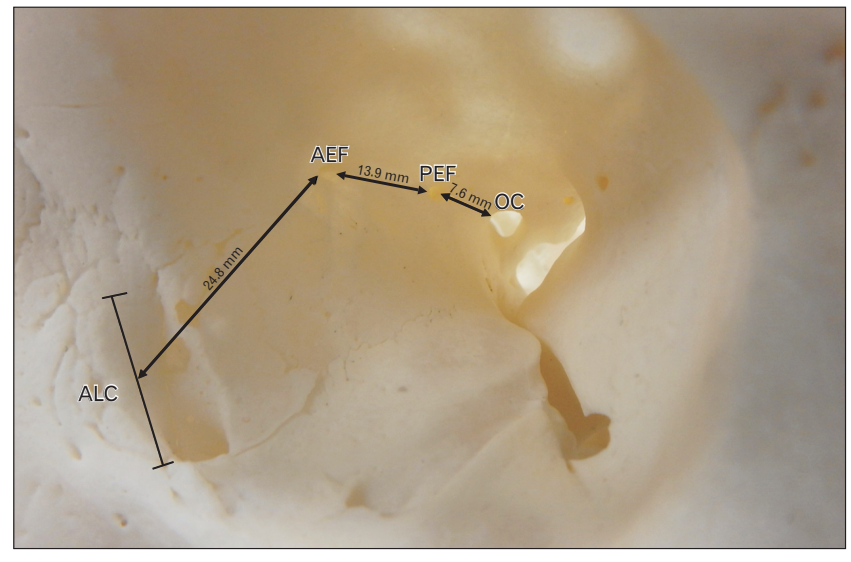

Fig. 6. Recommended surgical guideline. Based on the data from the Hispanic and European populations, the recommended surgical guideline between the ALC, AEF, PEF, and OC is 24.8-13.9-7.6 mm, respectively. Left orbit. AEF, anterior ethmoidal foramen; ALC, anterior lacrimal crest; OC, optic canal; PEF, posterior ethmoidal foramen.

an AEF present. Comparatively, studies reported anywhere from $1.6 \%$ to $2.0 \%$ of orbits with a missing PEF $[1,2,4,10]$. Lastly, 3/79 orbits (3.8\%) presented with an 'accessory AEF' located anterior to the AEF. Often, this foramen did have a small structure traversing through it. Only one study conducted by Piagkou et al. [1] previously reported finding an 'accessory AEF' in $1.2 \%$ of their sample size.

\section{Discussion}

Previous literature has reported deviation from the '24- 
12-6 surgical guideline in various populations, however, this was the first study to examine the anatomy of the medial orbital wall in the Hispanic population.

The results of this study suggest there was a statistically significant difference $(P=0.0019, P<0.0001, P<0.0001$, respectively) between the anatomical relationships along the medial wall of the orbit in the cadavers studied $(\mathrm{n}=79)$ versus the '24-12-6' mm surgical guideline. The numerical guideline that best fit our data was '24.8-13.9-7.6' mm (Fig. 6).

Based on family-reported ethnicities, cadavers were divided into two groups: European $(n=70)$ and Hispanic $(n=9)$. The anatomical relationship between the ALC-AEF, AEFPEF, PEF-OC for European and the Hispanic samples are best described as '24.7-13.8-7.5' $\mathrm{mm}$ and '25.1-14.8-8.3' $\mathrm{mm}$ respectively, and these were both statistically significant from the '24-12-6' surgical guideline. There was no statistically significant difference in the medial orbital wall measurements between these two populations.

To our knowledge, this manuscript was the first to analyze correlations between medial orbital wall measurements. This was calculated to determine if one medial orbital wall distance could be used to approximate how short or long the remaining two medial orbital wall ratios may be. Understanding the ratio between the ALC-AEF, AEF-PEF, or PEFOC can help clinicians more accurately estimate distances while performing medial orbital wall surgeries. Our analysis shows that the AEF-PEF distances have a significantly strong negative correlation $(\mathrm{r}=-0.4907)$ with the PEF-OC distances. The correlation between the AEF-PEF distances and PEFOC distances is clinically relevant because patients with longer AEF-PEF distances are likely to have shorter PEF-OC distances. This new surgical guideline indicates surgeons should more carefully navigate this portion of the medial orbital wall since the optic nerve would be closer to the surgical field.

Analyzing interactions on medial orbital wall measurements through a two-way ANOVA determined if sexual dimorphism or side of the body had a statistically significant effect on the medial orbital wall measurements. For the ALCAEF measurement, sex had no interaction on the distance, but the side of the orbit did have an effect on the data, where the average ALC-AEF measurement in the left orbit $(25.18 \mathrm{~mm})$ was larger than that of the right orbit $(24.44 \mathrm{~mm})$. With regards to the AEF-PEF measurement, the side of the body did not have any effect on the data, but sex had an effect on this measurement, where the average male AEF-PEF measurement
$(14.68 \mathrm{~mm})$ was larger than that of females $(13.30 \mathrm{~mm})$. Lastly, neither sex nor side of the face had a statistically significant effect on the PEF-OC measurement. There is no readily available explanation for these results; it is unclear why sexual dimorphism or side of the face may affect one measurement and not others. While it may be an anomaly, more research is needed to determine an explanation.

Surgeons should be aware that for each medial wall relationship, over $20 \%$ of the subjects studied had measurements that fell well below the surgical guideline: $27.8 \%(22 / 79)$ of orbits fell below the ALC-AEF surgical guideline $(24 \mathrm{~mm})$, $24.5 \%(19 / 79)$ of orbits fell below the AEF-PEF surgical guideline $(12 \mathrm{~mm})$, and $20.5 \%$ (16/79) of orbits fell below the PEF-OC surgical guideline $(6 \mathrm{~mm})$. The minimum distances for each respective measurement was $19.5 \mathrm{~mm}, 6.0 \mathrm{~mm}$, and $3.5 \mathrm{~mm}$. Clinically, patients with medial orbital wall measurements located below the surgical guideline are at the highest risk of experiencing surgical complications since the surgeon would not yet be expecting to encounter the structures traversing the foramen.

Our findings agree with the previously published literature: there is variability in medial orbital wall measurements between populations and the ' $24-12-6$ ' $\mathrm{mm}$ guideline is not ubiquitously applicable. For our study, the European medial orbital wall measurements followed a ' $25-14-8$ ' $\mathrm{mm}$ ratio and the Hispanic sample followed a '25-15-8' mm ratio. Other studies have reported various ratios dependent on ethnicity; African American: '25-14-6' mm [8], Caucasian: '24-14-6' mm [8], British Caucasian: '26-14-12'mm [4], Greek: '23-10-4' mm [1], Chinese: '25-11-6' mm [6], and Korean: '21-11-9' mm [7]. As these studies illustrate, there is significant variability in the medial orbital wall measurements between different populations and against the surgical guideline.

These findings are clinically relevant as understanding these skeletal relationships are critical for surgeons to preserve the structures traversing the foramina. Our findings suggest that the '24-12-6' mm surgical guideline did not apply to either population and should, therefore, be modified. Over $20 \%$ of the cadaver sample had ALC-AEF, AEF-PEF, or PEF-OC measurements that fell below the '24-12-6' mm surgical guideline. If a surgeon operated on any individual within this subset of our sample, special care is needed since the medial orbital wall measurements are shorter than expected. This is especially true for the PEF-OC measurement since the $\mathrm{OC}$ and the optic nerve could potentially be as close as $3.5 \mathrm{~mm}$ away from the posterior ethmoidal foramen; dam- 
age to the optic nerve may result in blindness.

A limitation of the study, and a common limitation across the current literature, is the reliance on family reported ethnicity. Family reported ethnicity lacks genetic testing information. Age of the cadavers was also a limitation of this study. The cadavers in the Body Donation Program were of an older age demographic, with the age range for those in this study being 52-98 and the average age of cadavers being 81 years old. Several aging processes that affect skeletal features, like bone absorption or bone remodeling, may have affected the measurements taken in this study. This is a standing limitation of this study common across most of the current literature. Despite these limitations, this study significantly contributes to the current body of literature by being the first to thoroughly investigate the anatomy of the medial orbital and the first to investigate these relationships in the South Texas Hispanic population.

\section{ORCID}

Kathylin M. Hester:

https://orcid.org/0000-0002-9943-097X

Omid B. Rahimi: https://orcid.org/0000-0002-0739-4132

Constance L. Fry: https://orcid.org/0000-0001-8787-0487

Haley L. Nation: https://orcid.org/0000-0002-9510-1167

\section{Author Contributions}

Conceptualization: HLN. Data acquisition: KMH, CLF, HLN. Data analysis or interpretation: KMH, OBR, CLF, HLN. Drafting of the manuscript: KMH, HLN. Critical revision of the manuscript: OBR, CLF, HLN. Approval of the final version of the manuscript: all authors.

\section{Conflicts of Interest}

No potential conflict of interest relevant to this article was reported.

\section{Acknowledgements}

The authors would like to thank the body donors for their contributions to education and research.

\section{References}

1. Piagkou M, Skotsimara G, Dalaka A, Kanioura E, Korentzelou V, Skotsimara A, Piagkos G, Johnson EO. Bony landmarks of the medial orbital wall: an anatomical study of ethmoidal foramina. Clin Anat 2014;27:570-7.

2. Rontal E, Rontal M, Guilford FT. Surgical anatomy of the orbit. Ann Otol Rhinol Laryngol 1979;88(3 Pt 1):382-6.

3. Lander MI, Terry O. The posterior ethmoid artery in severe epistaxis. Otolaryngol Head Neck Surg 1992;106:101-3.

4. Abed SF, Shams P, Shen S, Adds PJ, Uddin JM. A cadaveric study of ethmoidal foramina variation and its surgical significance in Caucasians. Br J Ophthalmol 2012;96:118-21.

5. Agthong S, Huanmanop T, Chentanez V. Anatomical variations of the supraorbital, infraorbital, and mental foramina related to gender and side. J Oral Maxillofac Surg 2005;63:8004.

6. Cheng AC, Lucas PW, Yuen HK, Lam DS, So KF. Surgical anatomy of the Chinese orbit. Ophthalmic Plast Reconstr Surg 2008;24:136-41.

7. Hwang K, Baik SH. Surgical anatomy of the orbit of Korean adults. J Craniofac Surg 1999;10:129-34.

8. Mehta MP, Perry JD. Medial orbital wall landmarks in three different North American populations. Orbit 2015;34:72-8.

9. Passel JS, Cohn DV, Lopez MH. Census 2010: 50 million Latinos. Hispanics account for more than half of nation's growth in past decade. [Internet]. Washington, D.C.: Pew Hispanic Center; 2011 [cited 2020 Aug 25]. Available from: https://www. pewresearch.org/hispanic/2011/03/24/hispanics-account-formore-than-half-of-nations-growth-in-past-decade/.

10. Caliot P, Plessis JL, Midy D, Poirier M, Ha JC. The intraorbital arrangement of the anterior and posterior ethmoidal foramina. Surg Radiol Anat 1995;17:29-33. 